\title{
Supplementary Information for Strong Electric Field Observed at the Interface of Aqueous Microdroplets
}

\author{
Hanqing Xiong ${ }^{1}$, Jae Kyoo Lee ${ }^{2}$, Richard N. Zare ${ }^{2, *}$, Wei Min ${ }^{1, *}$ \\ ${ }^{1}$ Department of Chemistry, Columbia University, New York, NY 10027 \\ ${ }^{2}$ Department of Chemistry, Stanford University, Stanford, CA 94305 \\ Corresponding authors: zare@ stanford.edu or wm2256@ columbia.edu
}




\section{Experimental methods}

Superhydrophobic coverslip preparation: First, standard coverslips $(22 \mathrm{~mm} \times 22 \mathrm{~mm}$ square, $0.17-\mathrm{mm}$ thick) were washed and hydroxylated in Piranha solution $\left(\mathrm{v}\left(\mathrm{H}_{2} \mathrm{SO}_{4}\right): \mathrm{v}\left(\mathrm{H}_{2} \mathrm{O}_{2}\right)=3: 1\right.$, $90{ }^{\circ} \mathrm{C}$ overnight), and further rinsed by 30 -min ultrasonic cleaning in deionized water for more than 3 times. The coverslips were then dried and soaked in a glass vessel filled with $10-\mathrm{mL}$ toluene, 0.1-mL methyltrichlorosilane (M85301, Sigma), and 20- $\mu$ L hydrochloric acid at $4{ }^{\circ} \mathrm{C}$ for about 10 hours. ${ }^{1}$ After washed in toluene for three times and rinsed by deionized water (and dried), the coverslips are ready to be used.

Microdroplets preparation: Hexadecane (52209, Sigma), silicone oil (Fisher S159500 and Sigma 378348), and water (38796, Sigma) were directly used in the experiment without further purification. $0.15-\mathrm{mL}$ charged dye water solution with the corresponding concentration was mixed with 0.4-mL silicone oil or hexadecane, and further sonicated in a standard centrifuge tube until the upper layer was opaque; then $30-\mu \mathrm{L}$ upper layer of the mixture was quickly transferred into the chamber of imaging spacer attached on a superhydrophobic coverslip, and then carefully sealed by a glass slide. Make sure the coverslip is always at the bottom. Wait for $10 \mathrm{~min}$ so that the droplet has sunk onto the coverslip; then the sample is ready for imaging. For samples used in Figure $\mathbf{3 ~ c}$, 0.1-M HCl (339253, Sigma) solution has been used to prepare microdroplets. Samples were measured after keeping in room temperature for 4 hours, which resulted in minimum variation from droplets to droplets.

Instrument design of two-photon fluorescence and SREF imaging: As shown in Figure S2, the PicoEmerald S system (Applied Physics \& Electronic, Inc.) was used as the laser source for all imaging excitation. It has two pulsed laser beam outputs: one is an IR beam fixed at $1031.8 \mathrm{~nm}$; the other one is the tunable OPO signal pumped by the second harmonic generation of the IR beam. The pulses from these two beams are temporally synchronized. All of them have a pulse width $\sim 2$ ps, FWHM bandwidth $\sim 0.6 \mathrm{~nm}$. The two beams are separately expanded; the OPO signal passed through a delay line, and the IR beam passed through a 20-MHz resonance electro-optic modulator; the two beams are then combined and sent to an inverted microscope (IX71, Olympus) configured with a home-built 2-D galvanometer scanner (GVSM002, Thorlabs). An achromatic half-wave plate was added into the light path of the combined beam to control the polarization of the beams. The scan lens (SL50-CLS2, Thorlabs) and tube lens (TL200-CLS2, Thorlabs) enable another $4 \times$ expansion of the beam. Finally, the back pupil of the objective (UPLSAPO, 1.2 NA, Olympus) is overfilled. Besides the fast galvanometer scanner, a relatively slow piezo stage (P545, Physik Instrumente) scanner was also used for imaging with a much smaller pixel size.

There are two signal detection modules in this system. One is for stimulated Raman Scattering (SRS) imaging, which is only used to check the temporal overlap between two beams. Details about the configuration can be found in our previous publication. ${ }^{2}$ The other signal detection module is for fluorescence imaging, which includes two-photon fluorescence imaging and SREF imaging. The fluorescence signal is detected in the backward direction. After passing through the dichroic mirror (FF825-SDi01), besides the fixed filter set (FF01-709/167-25, Semrock) used to block the laser beam, the backward-collected fluorescence will meet another filter set (FF01- 
$747 / 33$, Semrock). After this, the fluorescence is coupled into a multimode fiber and sent to a high quantum yield $(\sim 70 \%$ at $700 \mathrm{~nm})$ signal photon counting module (SPCM-NIR-14-FC, 70-cps dark counts, Excelitas). The output TTL pulse of the detector is sent into the counter channel of a NI card (PCI-6259, NI) for signal processing. For all SREF imaging, both two beams are used. For two-photon imaging with the 1031.8-nm IR pulse, the OPO signal beam can be blocked. All scanning driving, data collection, and their synchronization are achieved by the NI card (PCI-6259, NI) with an in-house-written LabVIEW program.

Two-photon fluorescence imaging and SREF imaging of microdroplet in oil: For two-photon imaging with the 1031.8-nm IR pulse, the OPO signal beam can be blocked. For all hyperspectral SREF imaging, nine images at different wavenumbers are recorded sequentially as the pump laser swept across the resonance of Rhodamine 800 (Rh800) nitrile mode. For SREF imaging of microdroplets (especially for microdroplet prepared at low concentration $(50 \mathrm{nM})$ ), to avoid laserinduced bleaching, the pixel dwell time was set to $20 \mu$ s per pixel and the pixel size was set to 200 $\mathrm{nm}$, and a 40-frame average was used to enable a good signal-to-noise ratio for the imaging at each wavenumber. The laser powers were set to $10 \mathrm{~mW}$ for the pump beam, and $11 \mathrm{~mW}$ for the Stokes beam. For each field of view of microdroplets, more than two independent measurements are made to double-check the Raman peak measurements, which helped to remove errors induced by laser tuning instability.

Data analysis: For each droplet prepared by 50-nM Rh800 solution (Fig. 2e-g, Fig. S3), because $\mathrm{Rh} 800$ molecules in the interior region are depleted, all the signal comes from the interface. Therefore, the summation of all the fluorescence counts at each wavenumber corresponds to one measurement point on the SREF spectrum. The bulk reference is measured in the $20-\mu \mathrm{M} \mathrm{Rh} 800$ water solution. Both $\mathrm{pH}=7$ (Figs. 2f,g) and $\mathrm{pH}=1(\mathrm{pH}$ tuned by $\mathrm{HCl}$, Fig. 3c) are measured, and the SREF peak are the same. And all the system configurations for bulk measurements is the same as that for microdroplet measurement.

For each droplet prepared by the $20-\mu \mathrm{M}$ Rh800 solution (Fig. 2b), both the interior and the interface have detectable signals. The summations of all the fluorescence counts in the interior and the interface at each wavenumber are used as measurement points of SREF spectra for corresponding regions, respectively (dots in Fig. 2c). After this, the raw nine-point spectra of the $\mathrm{Rh} 800$ nitrile mode for corresponding droplets or regions are achieved. The raw SREF spectrum is a combination of pure SREF signal, two-photon fluorescence background, and anti-Stokes fluorescence background ${ }^{2}$, which can be accurately fit by the summation of a Voigt line shape (the line shape of the pure SREF signal) and an exponential decay (a combination effect of Boltzmann distribution and time accumulated bleaching for the fluorescence backgrounds). As shown below,

$$
f(v)=x_{1} \cdot e^{x_{2} \cdot(v-\text { const })}+x_{3} \cdot \operatorname{Re}[\omega(z)]
$$

where the first term represents the overall background, and the second term represents the pure SREF signal (as shown in Figures 2 c,f and Figures S3b,d); const was set to $2264 \mathrm{~cm}^{-1}$ for calculation convenient; $\operatorname{Re}[\omega(\mathrm{z})]$ is the real part of the Faddeeva function ${ }^{3}$ with $z=\frac{\left(v-x_{4}\right)+i \frac{x_{5}}{2}}{9 \mathrm{~cm}^{-1} / 2 \sqrt{\ln 2}}$ (here $9 \mathrm{~cm}^{-1}$ is our laser linewidth). Fitting the $f(v)$ to the nine-point raw spectral data provides the five parameters $x_{1}$ to $x_{5}$. And $x_{4}$ is the peak position of the Rh800 nitrile mode. 

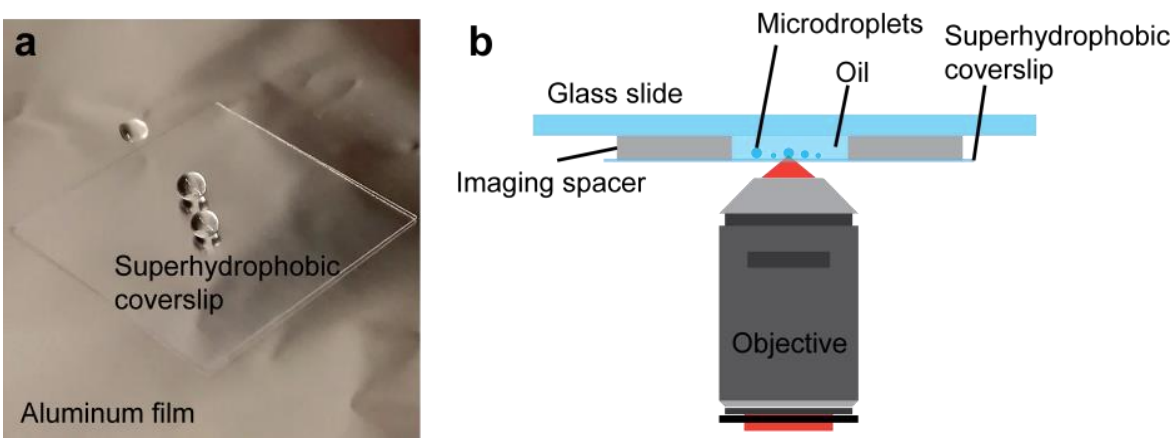

Figure S1. Sample preparation of the water microdroplets in oil. (a) A typical superhydrophobic coverslip used in this research. (b) The schematic of the microdroplet imaging setup. 


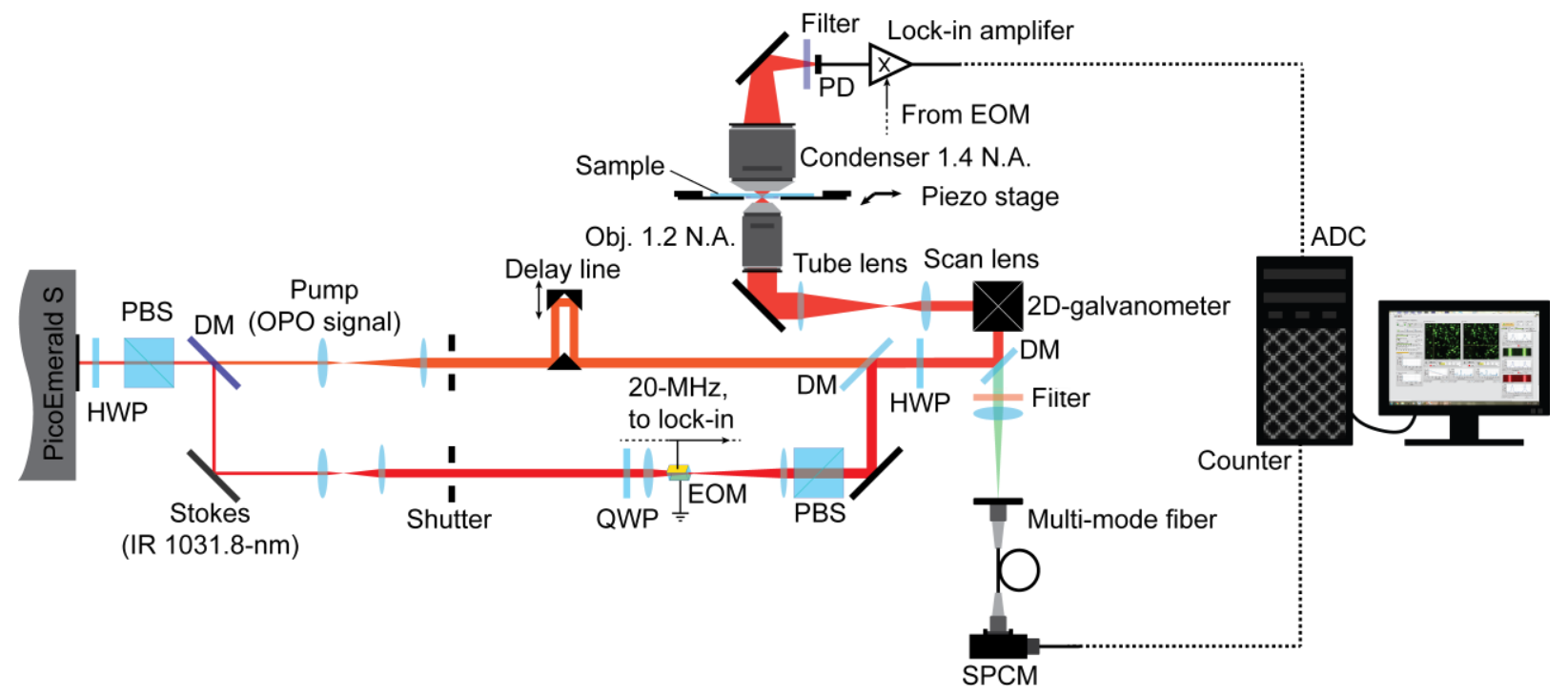

Figure S2. The home-built imaging system diagram, including two-photon fluorescence and stimulated Raman excited fluorescence (SREF) imaging. HWP, half-wave plate; PBS, polarized beam splitter; DM, dichroic mirror; QWP, quarter-wave plate; EOM, electro-optic modulator; SPCM, single photon counting module; ADC, analog-to-digital converter; PD, photodiode. 

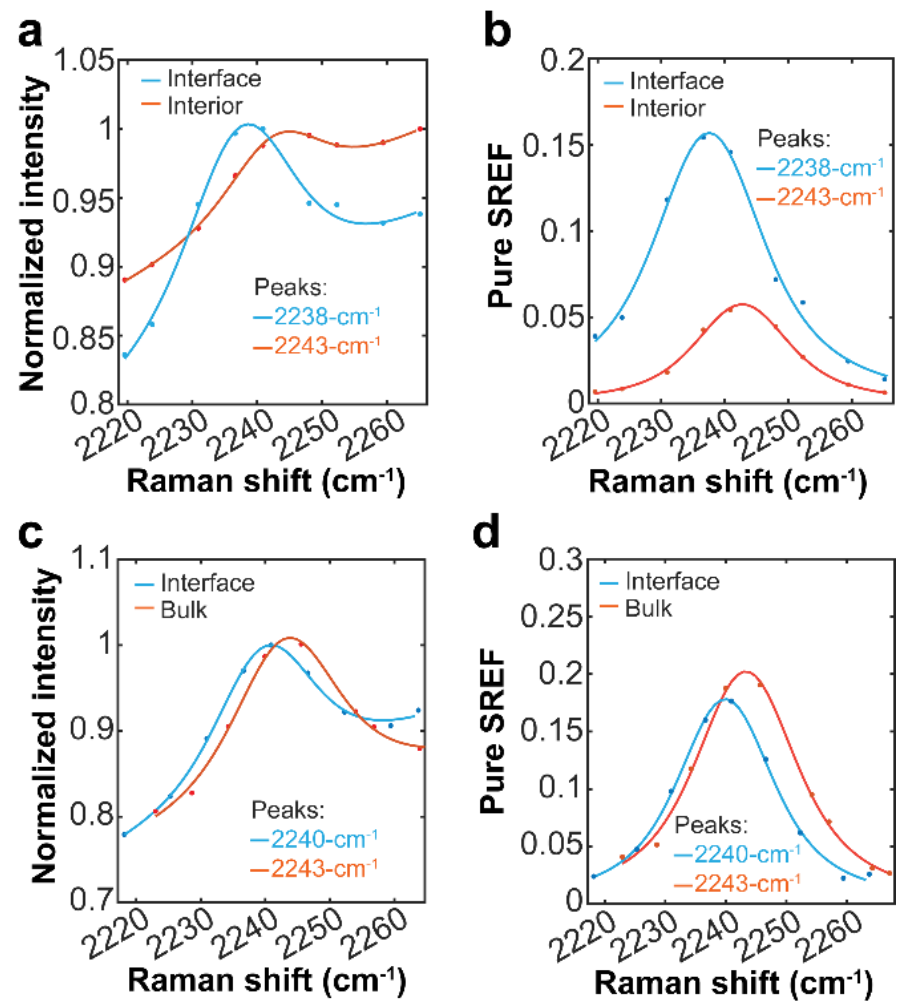

Figure S3. Raw SREF spectra ((a) and (c)) and background-subtracted (pure) SREF spectra ((b) and (d)) after subtracting the broadband background. Figure $2 \mathrm{c}$ is the normalized version of (b), and the corresponding raw SREF spectra are shown in (a); Figure $2 \mathrm{f}$ is the normalized version of (d), and the corresponding raw SREF spectra are shown in (c). 

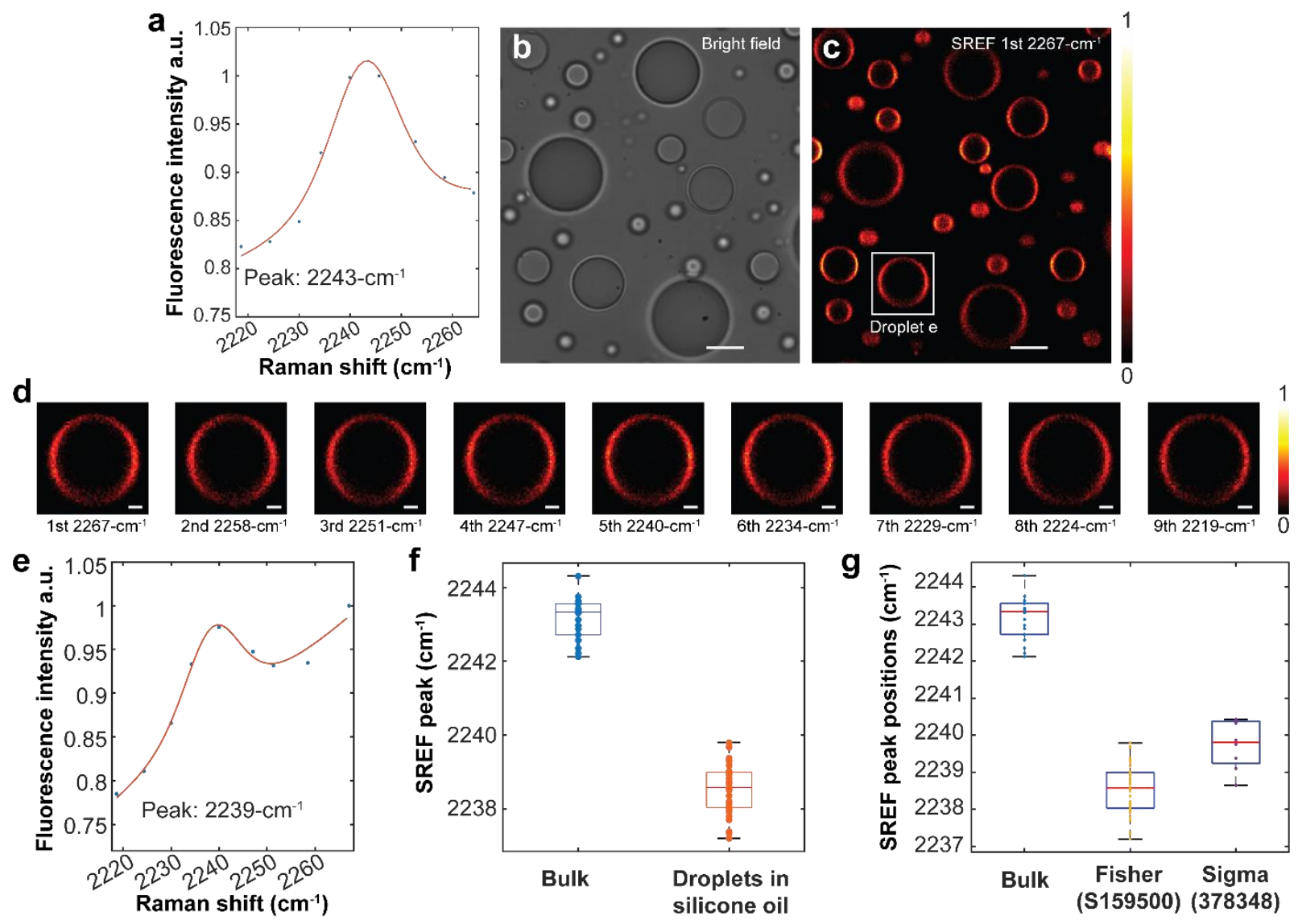

Figure S4. SREF imaging of water microdroplets in silicone oil. (a) A typical SREF spectrum of the Rh800 nitrile mode measured in the $20-\mu \mathrm{M}$ bulk water solution. (b) Bright-field imaging of the microdroplets of 50-nM Rh800 water solution in silicone oil and (c) the first frame of corresponding hyperspectral SREF imaging of the Rh800 nitrile mode. (d) The whole hyperspectral SREF image series of droplet e (as labeled on (c)) and (e) the corresponding SREF spectrum. (f) Box plot of the distribution of Rh800 nitrile peak positions for 18 measurements in bulk water and 38 measurements on droplets, respectively. Every droplet has been measured twice. $(\mathrm{g})$ Batches of silicone oil from two different vendors have been tested (Fisher (S159500) and Sigma (378348)). In (a) and (e), dots are measurements and curves are fitting results (detail in Experimental methods). Scale bar: $10 \mu \mathrm{m}$ in (b) and (c); $2 \mu \mathrm{m}$ in (d). 


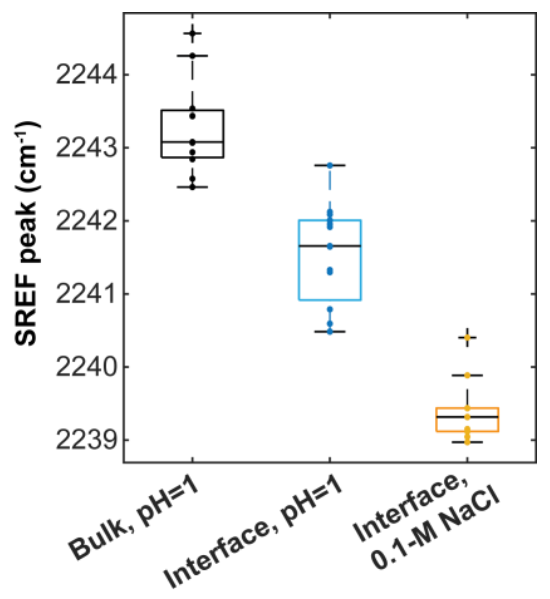

Figure S5. Box plot of SREF peaks measured in the bulk 0.1-M HCl solution $(\mathrm{pH}=1)$, the microdroplet interface in $0.1-\mathrm{M} \mathrm{HCl}$ solution, and the microdroplet interface in $0.1-\mathrm{M} \mathrm{NaCl}$ solution. $\mathrm{Rh} 800$ probes were dissolved in each solution at $10 \mu \mathrm{M}$ concentration. 


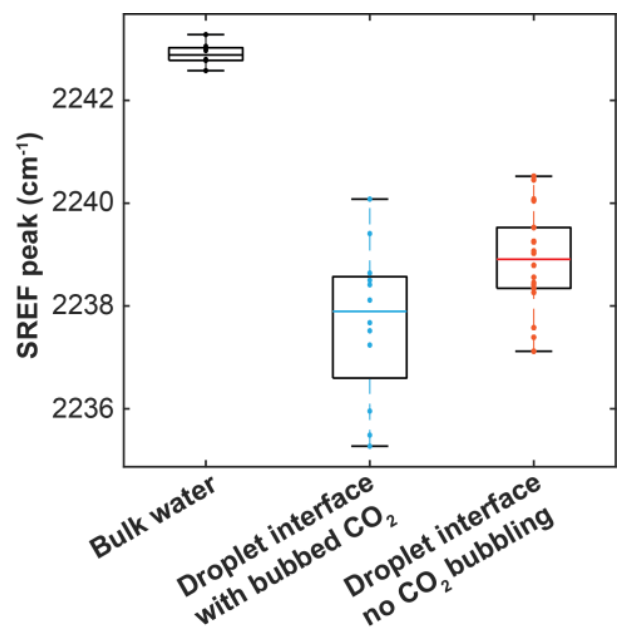

Figure S6. Box plot of SREF peaks of the Rh800 nitrile band measured in the bulk water (8 independent measurements), the interface of the microdroplet with $\mathrm{CO}_{2}$ bubbled for 1 min (12 independent measurements), and the interface of the microdroplet without $\mathrm{CO}_{2}$ bubbling (16 independent measurements as shown in Fig. 2d). Rh800 probes were dissolved in each solution at 20 $\mu \mathrm{M}$ concentration. 


\section{References}

1. Wong, J. X.; Yu, H.-Z. Preparation of transparent superhydrophobic glass slides: Demonstration of surface chemistry characteristics. Journal of Chemical Education 2013, 90 (9), 1203-1206.

2. Xiong, H.; Shi, L.; Wei, L.; Shen, Y.; Long, R.; Zhao, Z.; Min, W. Stimulated Raman excited fluorescence spectroscopy and imaging. Nature Photonics 2019, 13 (6), 412.

3. Abrarov, S. M.; Quine, B. M. Efficient algorithmic implementation of the Voigt/complex error function based on exponential series approximation. Applied Mathematics and Computation 2011, 218 (5), 1894-1902. 